\title{
Evidence for a First Order, Finite Temperature Phase Transition in 8 Flavor QCD
}

\author{
Xiao-Yong Jin* \\ Department of Physics, Columbia University, New York, NY 10027, USA \\ E-mail: xj2106@columbia.edu \\ Robert D. Mawhinney \\ Department of Physics, Columbia University, New York, NY 10027, USA \\ E-mail: rdmephysics.columbia.edu
}

\begin{abstract}
As part of our ongoing investigations of QCD with many flavors of quarks, here we report on studies of the finite temperature phase transition for eight-flavor QCD with the DBW2 gauge action and naïve staggered fermions. We find a clear first order phase transition between the chirally asymmetric phase at zero temperature and the chirally symmetric phase at finite temperature, signaled by a two-state signal for $\langle\bar{\psi} \psi\rangle$ at a non-zero temperature. We see this signal at a gauge coupling of $\beta=0.54$, where, to set the scale, the zero temperature value for $f_{\pi}$, in the chiral limit, is $0.06661(92)$. This strong, first-order signal is seen for two different values of the quark mass, $m_{q}=0.007$ and 0.0195 , at $N_{\tau}=8$ and 6 respectively. Using $f_{\pi}\left(m_{q}\right)$ as the scale, the critical temperature is measured to be $T_{c} / f_{\pi}=1.638(93)$ at $m_{\pi} / f_{\pi}=3.329(30)$ for $m_{q}=0.007$, and $T_{c} / f_{\pi}=1.779(27)$ at $m_{\pi} / f_{\pi}=4.093(15)$ for $m_{q}=0.0195$. At a weaker coupling $\beta=0.56$, where at zero temperature and in the chiral limit we find $f_{\pi}=0.0312(10)$, the first order signal becomes numerically invisible to us for the $N_{\tau} \leq 14$ lattices we have investigated so far.
\end{abstract}

The XXVIII International Symposium on Lattice Filed Theory

June 14-19,2010

Villasimius, Sardinia Italy

\footnotetext{
* Speaker.
} 

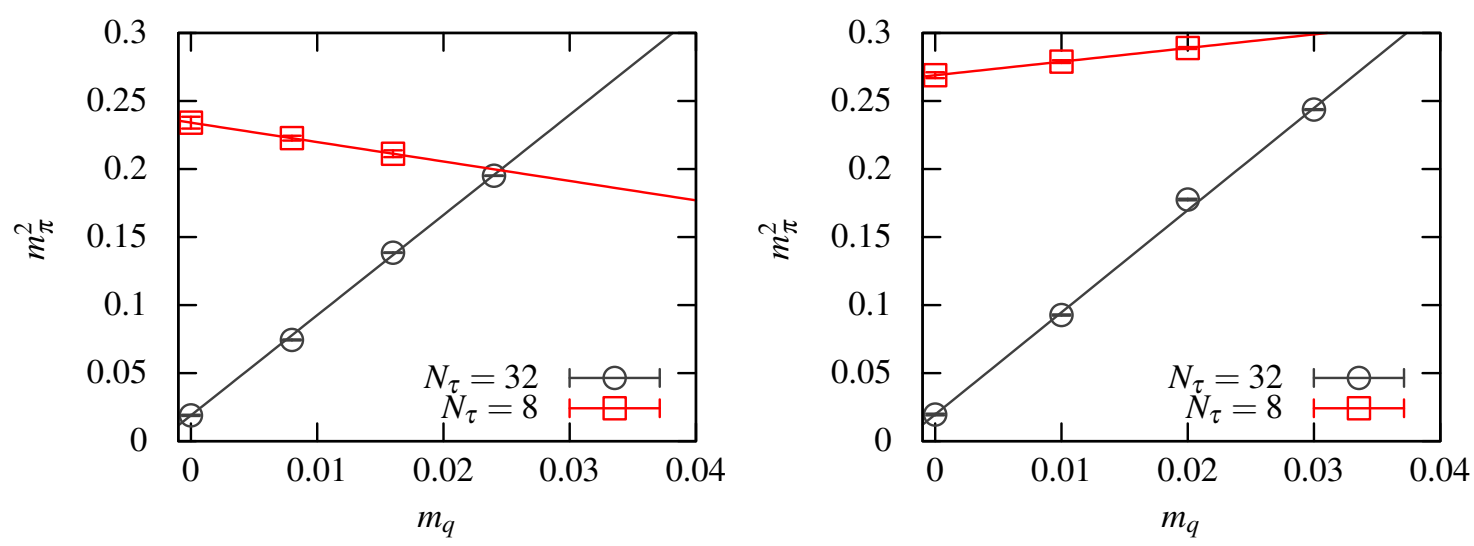

Figure 1: Pion screening masses at finite temperature $\left(N_{\tau}=8\right)$ compared to pion masses at zero temperature $\left(N_{\tau}=32\right)$. The left panel shows 8 flavors at $\beta=0.56$; the right panel shows 12 flavors at $\beta=0.49$.

\section{Introduction}

This work is a continuation of our ongoing studies of many flavor QCD, as reported in previous conferences $[1,2]$. We use the DBW2 gauge action and naïve staggered fermion action with the exact RHMC algorithm. We have studied a series of bare coupling values and quark masses for both 8 and 12 flavors at zero temperature $\left(N_{\tau}=32\right)$. Various hadronic observables have been measured on the lattices, to determine both the general lattice scale and the phase of the theory. Our conclusion is that QCD with both 8 and 12 flavors exhibits properties that are consistent with spontaneous chiral symmetry breaking at zero temperature. We have also studied the finite temperature behavior of the $N_{f}=8$ and 12 flavor QCD at $N_{\tau}=8$ [2]. A notable result is the behavior of the mass of the pion shown in Figure 1. At zero temperature, we find $m_{\pi}^{2}=B m_{q}$ for both 8 and 12 flavors, which is the expected behavior for a Goldstone boson produced by spontaneous chiral symmetry breaking. Such behavior goes away at finite temperature, where chiral symmetry is restored, as is clearly evident in the figure. Measurements of Wilson loops along the temporal direction, shown in Figure 2, support the existence of a finite temperature phase by clearly showing a nonzero value at $N_{\tau}=8$.

The chiral symmetry restoring finite temperature phase transition in QCD is expected to be first order [3], when the number of massless fermions is equal to or larger than 3. Since we have established that chiral symmetry is spontaneously broken at zero temperature and seen that it is restored at finite temperature, we wanted to see if the transition is first order for a range of non-zero quark masses accessible with current computer resources. If the transition is strongly first order, one should see the discontinuity of the order parameter manifest itself in co-existing, metastable evolutions of the lattice for precisely chosen parameters on large volumes. Non-zero fermion masses are expected to weaken any first order signal, and, for large enough masses, the phase transition will end in a second order critical point and then become a crossover. If a first order, finite temperature phase transition is found for non-zero quark masses, one can be fairly confident that this will persist as the quark masses go to zero.

In our previous, zero temperature work, we have seen a rapid change in the lattice scale with the coupling for 8 flavors [1] and a much more significant change for 12 flavors [2]. To investigate 

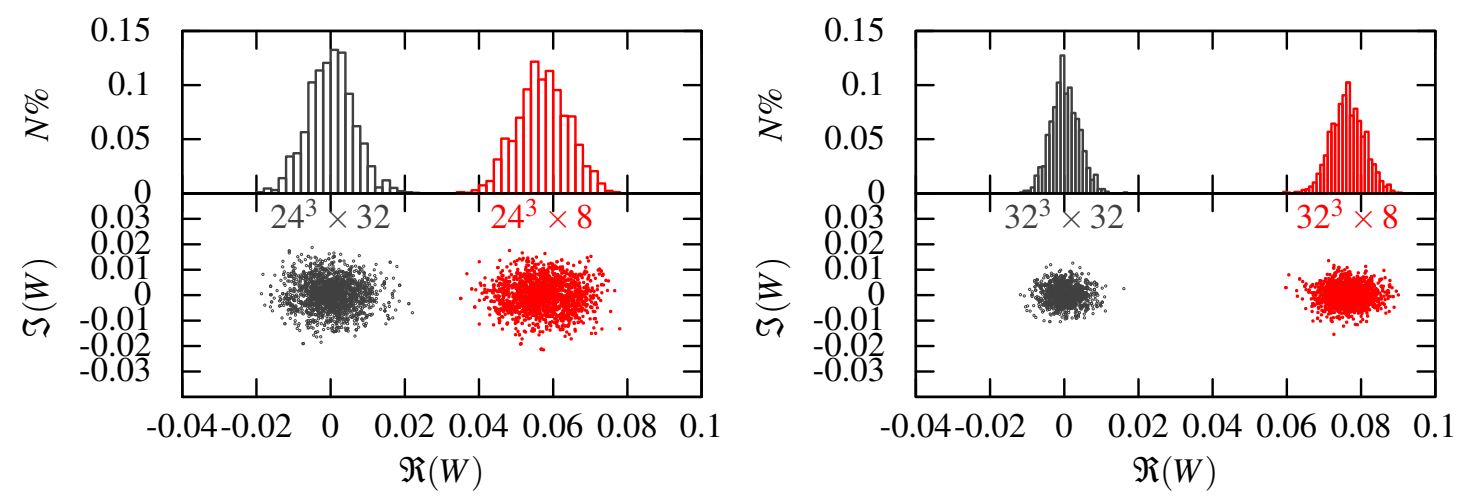

Figure 2: Values for Wilson loops along the temporal direction at finite temperature $\left(N_{\tau}=8\right)$ compared to the ones at zero temperature $\left(N_{\tau}=32\right)$. The lower part of each figure shows a scatter plot of the real (horizontal axis) and imaginary parts (vertical axis) of the Wilson loops, while the upper part shows a histogram of the real part of the Wilson loops. The histogram is normalized such that the height of each block represents the percentage of numbers in the bin. The left figure shows 8 flavors with $m_{q}=0.008$ at $\beta=0.56$; the right shows 12 flavors with $m_{q}=0.01$ at $\beta=0.49$. Lattice sizes are labeled in the figures.

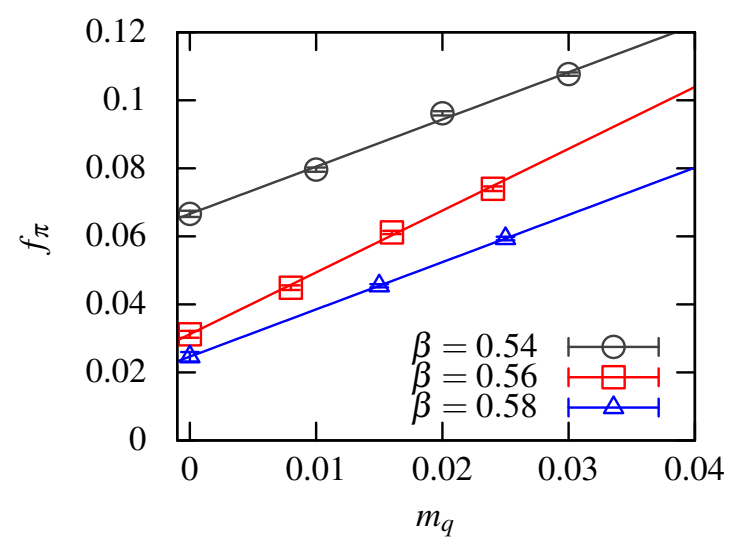

Figure 3: The pion decay constant, $f_{\pi}$, for 8 flavors at zero temperature, with a linear extrapolation to the chiral limit.

the details of the finite temperature phase transition and to interpret it with physical quantities, it is beneficial for us to use $\beta$ values where we have zero temperature ensembles. We can then probe various discrete values of temperature or $N_{\tau}$ by continuously tuning the bare quark mass as an input. We chose $\langle\bar{\psi} \psi\rangle$ to be the order parameter and ran simulations starting with both ordered and disordered gauge fields. A discontinuity due to a first order phase transition should result in two co-existing, metastable thermalized lattice ensembles with the same set of input parameters and having different values of $\langle\bar{\psi} \psi\rangle$.

\section{Finite temperature phase transition with 8 flavors}

In Figure 3 we show a linear extrapolation to the chiral limit of $f_{\pi}$ for three $\beta$ values where we have extensive zero temperature results for 8 flavors. One sees that between $\beta=0.54$ and 0.56 , 

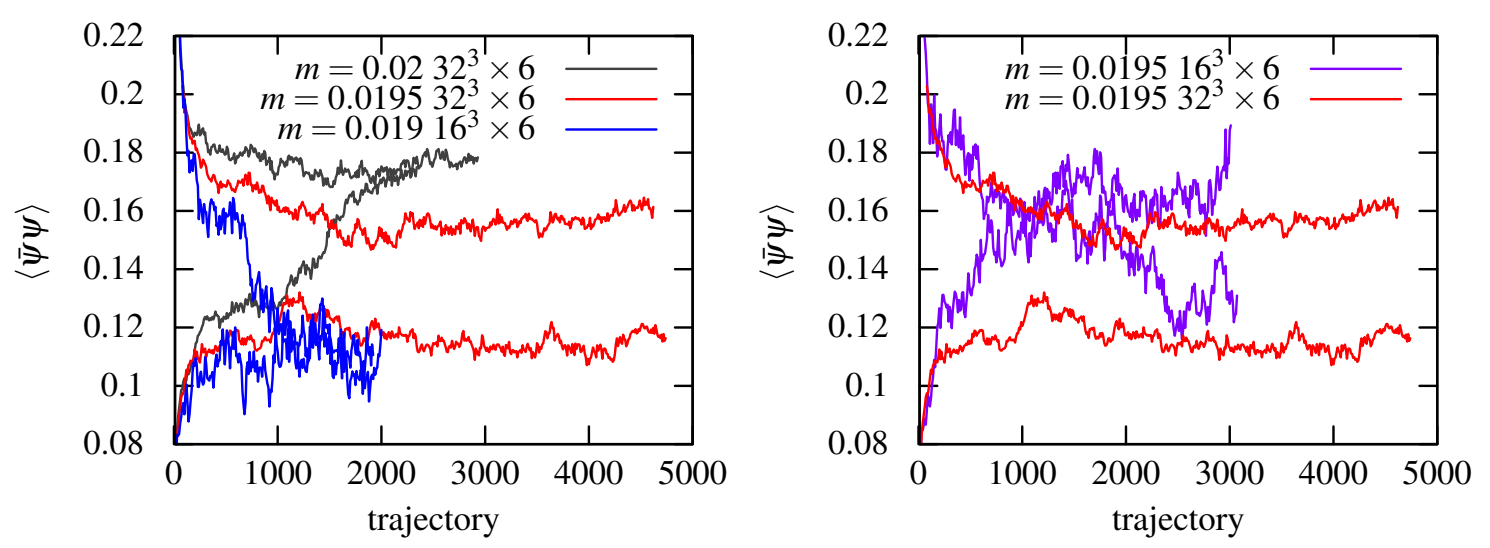

Figure 4: The evolution of $\langle\bar{\psi} \psi\rangle$, with both ordered (curves starting from bottom) and disordered starts (curves starting from top), using 8 flavors at $\beta=0.54$ and $N_{\tau}=6$. The left panel shows the transition for 3 different masses. The right panel shows 2 different lattice volumes at the transition mass, $m_{q}=0.0195$.
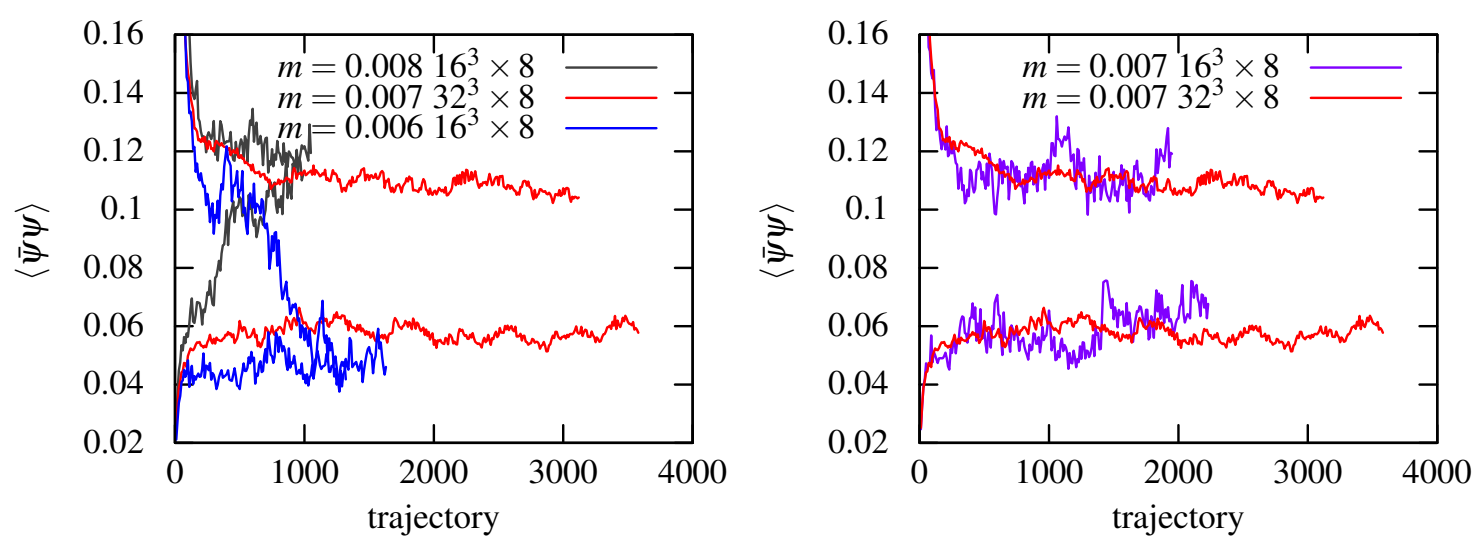

Figure 5: The evolution of $\langle\bar{\psi} \psi\rangle$ with both ordered (curve starting from bottom) and disordered starts (curve starting from top), using 8 flavors at $\beta=0.54$ and $N_{\tau}=8$. The left panel shows the transition for 3 different masses. The right panel shows 2 different lattice volumes at the transition mass, $m_{q}=0.007$.

the lattice scale changes by almost a factor of 3 . We expect the first order signal to be the strongest at small masses and the strongest coupling, $\beta=0.54$, so we have begun our simulations there.

Figure 4 shows the evolution of $\langle\bar{\psi} \psi\rangle$ at $\beta=0.54$ and $N_{\tau}=6$. By running with different quark masses, a metastability for nearly 4000 trajectories is seen at $m_{q}=0.0195$ with a lattice size of $32^{3} \times 6$. We find a discontinuity of $\Delta\langle\bar{\psi} \psi\rangle=0.0416(20)$ at this quark mass. Metastability is very sensitive to the quark mass; we have to tune the quark mass to within $3 \%$ to see two co-existing states. A large spatial volume, $32^{3}$ is also required, as shown in the right panel of Figure 4, where the metastability is not apparent on a $16^{3} \times 6$ volume.

For $N_{\tau}=8$, shown in Figure 5, metastability is seen at a much smaller quark mass, $m_{q}=0.007$, for more than 2000 trajectories. The first order signal is stronger, with a discontinuity $\Delta\langle\bar{\psi} \psi\rangle=$ $0.0508(17)$. The quark mass is tuned within $15 \%$. And both $16^{3}$ and $32^{3}$ volumes are clearly metastable.

We can draw the chiral symmetry restoration transition diagram for 8 flavors at $\beta=0.54$ as 

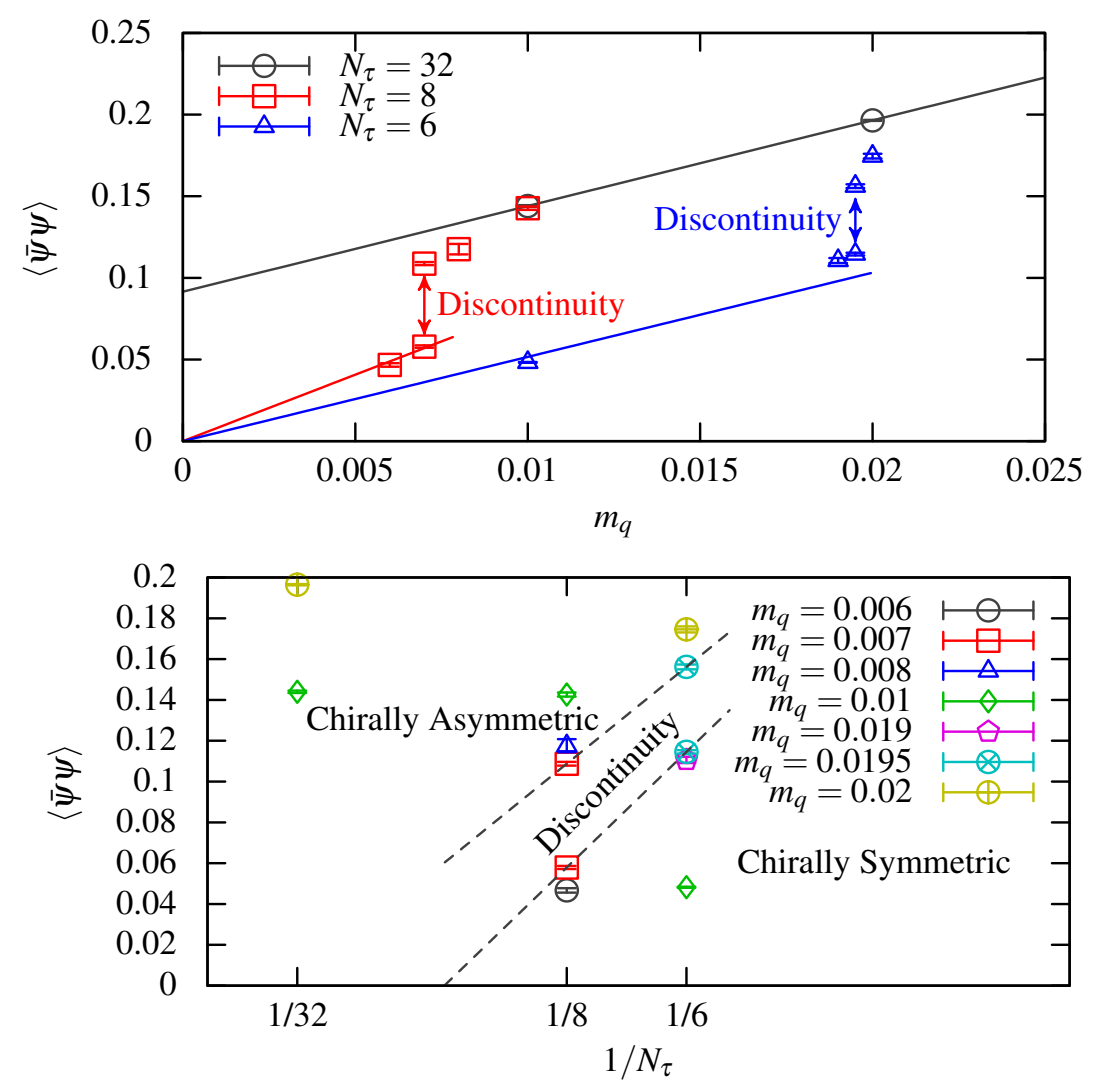

Figure 6: A diagram showing the values of $\langle\bar{\psi} \psi\rangle$ at $\beta=0.54$ with the discontinuity due to the first order phase transition explicitly shown. The upper panel shows $\langle\bar{\psi} \psi\rangle$ versus $m_{q}$ and the lower panel shows $\langle\bar{\psi} \psi\rangle$ versus temperature $\left(1 / N_{\tau}\right)$.

shown in Figure 6. The upper panel shows the value of $\langle\bar{\psi} \psi\rangle$ at different temperatures while varying the quark mass. The top line shows a linear extrapolation of $\langle\bar{\psi} \psi\rangle$ at $N_{\tau}=32$ to the chiral limit. The discontinuity separates the chirally broken phase (upper points) and the chirally symmetric phase (lower points). The two lower lines, for $N_{\tau}=8$ and $N_{\tau}=6$, which have slopes of 8.14 and 5.16 respectively, are linear fits to $\langle\bar{\psi} \psi\rangle$ in the chirally symmetric phase. They are forced to go to the origin, which is the expected behavior of $\langle\bar{\psi} \psi\rangle$ at finite temperature. The fit at $N_{\tau}=6$ has a much larger $\chi^{2} /$ dof (about 260) than the fit at $N_{\tau}=8$ (about 5). This can be seen as a sign of weakened first order signal due to the relatively large fermion mass. The lower panel of Figure 6 is another way of visualizing the phase diagram. The two dashed lines are linear fits to the metastable signal of $\langle\bar{\psi} \psi\rangle$ at the edge of chirally broken phase (upper left region), which has a slope of 1.14, and the edge of chirally symmetric phase (lower right region), which has a slope of 1.36. In between is the inaccessible discontinuity region of $\langle\bar{\psi} \psi\rangle$.

Table 1 displays the critical temperature, $T_{c}\left(m_{q}\right)$, in units of $f_{\pi}\left(m_{q}\right)$. The values of $f_{\pi}\left(m_{q}\right)$ and $m_{\pi}\left(m_{q}\right)$ come from naïve extrapolations of zero temperature results, where $m_{\pi}^{2} \propto m_{q}$ and $f_{\pi} \propto m_{q}$. The quoted errors of $m_{\pi} / f_{\pi}$ are only statistical errors. The errors quoted for $T_{c} / f_{\pi}$ uses error propagation from the error of the linear extrapolation of $f_{\pi}$ and the estimated error of $T_{c}$ that is described in the following. First, we can estimate the error in our determination of $m_{q}$ at the 


\begin{tabular}{r|l|l}
$m_{q}$ & 0.007 & 0.0195 \\
$m_{\pi}\left(m_{q}\right) / f_{\pi}\left(m_{q}\right)$ & $3.329(30)$ & $4.093(15)$ \\
\hline$T_{c}\left(m_{q}\right) / f_{\pi}\left(m_{q}\right)$ & $1.638(93)$ & $1.779(27)$
\end{tabular}

Table 1: The critical temperature, $T_{c}\left(m_{q}\right)$, of the first order, chiral symmetry restoring, finite temperature phase transition with 8 flavors, as observed at bare coupling $\beta=0.54$.

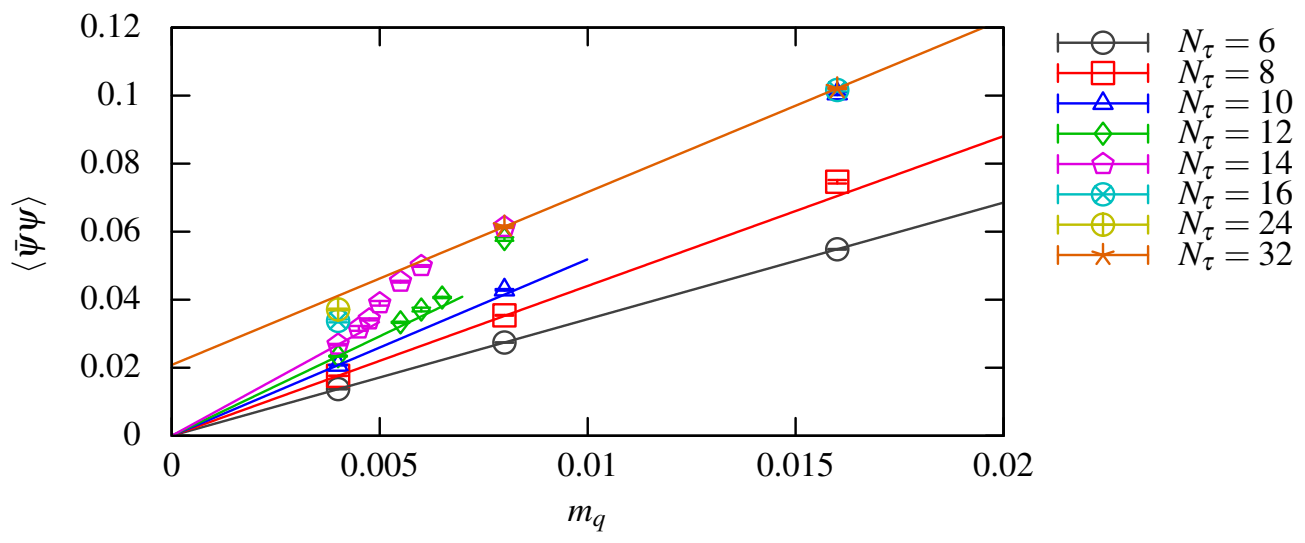

Figure 7: The values of $\langle\bar{\psi} \psi\rangle$ versus $m_{q}$ for 8 flavors at $\beta=0.56$. The first order transition point cannot be resolved numerically with our current simulations.

transition point from the range of quark masses which either do or do not show metastability. This gives us a quark mass uncertainty of 0.001 for $N_{\tau}=8$ and 0.0005 for $N_{\tau}=6$. At the edge of the chirally symmetric phase, using the two fits in the top panel of Figure 6, this uncertainty in $m_{q}$ can be translated to an uncertainty in $\langle\bar{\psi} \psi\rangle$ of 0.00814 and 0.00258 at $N_{\tau}=8$ and 6 respectively. These values can be further converted to an uncertainty on $T_{c}$ by the relation of $\langle\bar{\psi} \psi\rangle$ and $T_{c}$ at the edge of chirally symmetric phase which is represented by the lower dashed line in the lower panel of Figure 6. This procedure results in estimated errors on $T_{c}$ of 0.00599 and 0.00190 at $N_{\tau}=8$ and 6 respectively.

At weaker coupling, $\beta=0.56$, where the lattices are much finer than those at $\beta=0.54$, we did not find a clear signal of a first order transition. Figure 7 shows a survey of the parameter space we have covered. Among those points, for $m_{q} \leq 0.008$ and $N_{\tau} \neq 32$, the spatial volume of the lattices are $32^{3}$. Similar to the previous figure, the line at $N_{\tau}=32$ is a linear extrapolation of $\langle\bar{\psi} \psi\rangle$ at zero temperature. The lines drawn through the origin denote the $m_{q}$ dependence of $\langle\bar{\psi} \psi\rangle$ at finite temperature. As we are interested in a possible discontinuity of $\langle\bar{\psi} \psi\rangle$ we have done a careful scan, in quark mass, for $N_{\tau}=14$. Since we expect the discontinuity in $\langle\bar{\psi} \psi\rangle$ to be markedly smaller here than for $\beta=0.54$, we have focused on larger $N_{\tau}$ and smaller quark masses. Fixing $N_{\tau}=14$ and tuning the quark mass, we could not find a clear metastability signal like the ones seen at stronger coupling, $\beta=0.54$. The evolutions of $\langle\bar{\psi} \psi\rangle$ around $m_{q}=0.005$ at $N_{\tau}=14$ are shown in Figure 8 . In this figure, the evolution of $\langle\bar{\psi} \psi\rangle$ at $m_{q}=0.005$ is very noisy, similar to the one in Figure 4, at $\beta=0.54, m_{q}=0.007$ with a lattice size of $16^{3} \times 6$. It is a good indication that we are near the critical point of the transition, but either a much larger lattice spatial volume is required to produce metastable states or the quark mass is large enough so that the first order transition has almost 


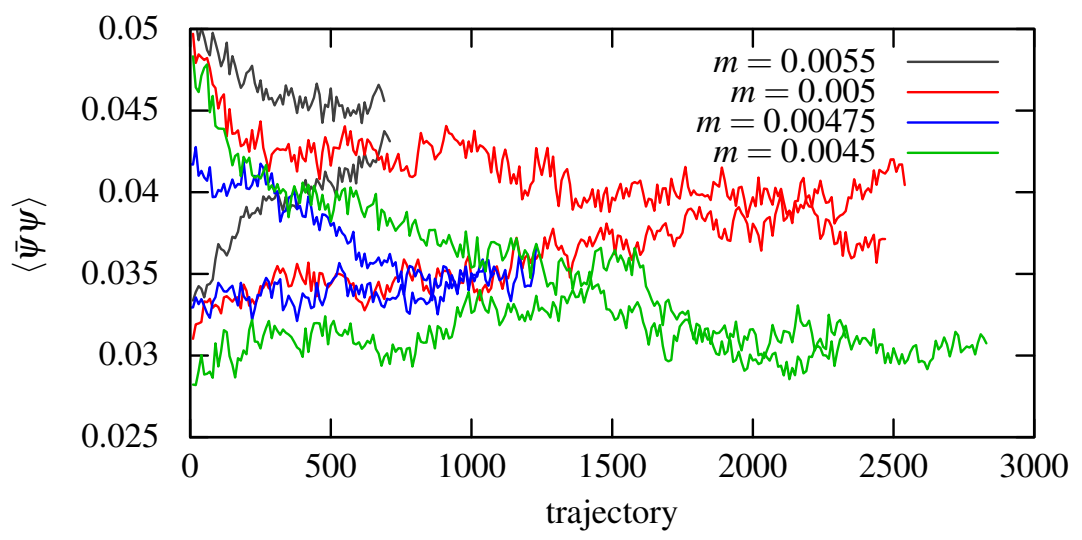

Figure 8: Evolutions of $\langle\bar{\psi} \psi\rangle$ with ordered and disordered starts at $N_{\tau}=14, \beta=0.56$.

weakened to become second order.

\section{Conclusions}

We have found a clear two-state signal in lattice simulations of QCD with 8 flavors at finite temperature, associated with the restoration of chiral symmetry, on lattices where the chiral limit value for $f_{\pi}=0.06661(92)$. The critical temperatures for two different pion masses, in units of the pion decay constant, were determined and are given in table 1. Extensive simulations at a weaker coupling, and lighter quark masses, have shown that the expected first order transition occurs for $N_{\tau}>14$. We have some evidence that $N_{\tau}=14$ is near the endpoint of the first order transitions. Much more computational time and careful tuning is required to search for the first order signal at these weaker couplings for 8 flavors. Further work needs to be done for 12 flavors to probe for the existence of first order thermal transition, which would give more evidence for the phase of the 12 flavor system at zero temperature.

We are thankful to all members of the RBC collaboration for useful discussions, suggestions and help with the CPS software used in this work. Our calculations were done on the QCDOC and NY Blue at BNL. This research utilized resources at the New York Center for Computational Sciences at Stony Brook University/Brookhaven National Laboratory which is supported by the U.S. Department, of Energy under Contract No. DE-FG02-92ER40699 and by the, State of New York.

\section{References}

[1] X.-Y. Jin and R. D. Mawhinney, Lattice QCD with Eight Degenerate Quark Flavors, PoS LATTICE2008 (2008) 059 [arXiv: 0812.0413 [hep-lat] ].

[2] X.-Y. Jin and R. D. Mawhinney, Lattice QCD with 8 and 12 degenerate quark flavors, PoS LAT2009 (2009) 049 [arXiv:0910.3216 [hep-lat]].

[3] R. D. Pisarski and F. Wilczek, Remarks on the Chiral Phase Transition in Chromodynamics, Phys. Rev. D29 (1984) 338-341. 